\title{
ORIGINAL
}

ARTICLES

$\square \square \square \square$

\section{Temporal Trends in Medical Student Burnout}

Maggie W. Hansell, MD; Ross M. Ungerleider, MD, MBA; Courtney A. Brooks, BA;

Mark P. Knudson, MD, MSPH; Julienne K. Kirk, PharmD; Jamie D. Ungerleider, PhD, MA, LCSW

BACKGROUND AND OBJECTIVES: There is a paucity of longitudinal data documenting the temporal development of distress and burnout during medical school. The aim of this study was to examine trends and identify stressors associated with medical student distress over 4 years of medical education.

METHODS: Medical students from the class of 2016 at a Liaison Committee on Medical Education-accredited medical school completed surveys nine times from orientation through after the residency match. Surveys included demographic variables and measured distress domains using the Medical Student Well-Being Index. The authors used Microsoft Excel to calculate the proportion of students screening positive for individual distress domains at each of the nine acquisition periods for descriptive analysis.

RESULTS: Students completed $\mathbf{8 8 6}$ total surveys for an $\mathbf{8 5 \%}$ response rate, which was relatively consistent across collection periods. Medical student distress and burnout increased from two (2\%) to $12(12 \%)$ respondents and from $19(17 \%)$ to 37 (38\%) respondents, respectively, from matriculation through after the residency match $(P<0.01)$. Depersonalization increased from $15(13 \%)$ to $34(35 \%)$ respondents and emotional exhaustion increased from six (5\%) to $22(22 \%)$ respondents across 4 years of medical education $(P<0.01)$. Emotional exhaustion peaked after medical school year 1, at 37 (45\%), and year 3 , at $45(44 \%)$ respondents, with improvement after summer break and residency match.

CONCLUSIONS: The results supported the literature demonstrating the development of burnout during medical school. Depersonalization increased early in the education process with minimal regression after development. Emotional exhaustion demonstrated a surprising increase after exposure to clinical clerkships. Further studies could support or refute the universality of these trends and evaluate prevention and intervention efforts targeting these key inflection points.

(Fam Med. 2019;51(5):399-404.)

doi: 10.22454/FamMed.2019.270753

$\mathbf{M}$ edical student distress comprises several domains, including burnout, depression, fatigue, stress, and quality of life (QOL), both mental and physical. ${ }^{1}$ Burnout is a multidimensional entity encompassing emotional

exhaustion (EE) along with altered response toward others (depersonalization [DP]) and toward self (reduced personal accomplishment). ${ }^{2}$ Associations exist between medical student burnout and decreased ethical fortitude (honesty and integrity) and empathy, as well as increased unprofessional behavior, risk of dropping out of medical school, and suicidal ideation. ${ }^{3}$

Medical students begin medical school with similar, if not better, mental health than their age-matched, college-educated counterparts, but mental health becomes worse relative to peers once medical school begins. ${ }^{4,5}$ Cross-sectional studies suggest that burnout is more prevalent in later years of medical school with depersonalization accounting for most of the increase. ${ }^{3}$ Several studies demonstrate that overall prevalence of burnout is nearly 50\% in students preparing to enter residency, suggesting that a great deal of burnout develops during medical school. ${ }^{6}$

Although personality characteristics, relationship status, life experiences outside of school, and coping strategies may impact propensity for burnout to varying degrees, these factors cannot independently explain the volume of distress that develops during medical school. ${ }^{3,7}$ By inference, the medical education process is at least partially responsible for the development of distress
From the Department of Family and Community Medicine (Drs Hansell, Knudson, and Kirk), Wake Forest University School of Medicine, Winston-Salem, NC (Ms Brooks); and Institute for Integrated Life Skills, Bermuda Run, NC (Drs R.M. Ungerleider and J.D. Ungerleider). 
domains in medical students. There is a paucity of longitudinal data demonstrating the trend in development of distress and burnout during medical school. This study aimed to investigate the temporal progression in medical student distress and its domains, including burnout, over the 4 years of medical education to inform future educational prevention and intervention efforts.

\section{Methods}

\section{Participants}

Starting in the fall of 2012 , we asked all matriculating medical students (120 students) at a Liaison Committee on Medical Education (LCME)-accredited medical school to participate by filling out a survey during orientation. We invited students from the class of 2016 to fill out paper surveys at eight subsequent group gatherings throughout medical school. Table 1 lists and describes data acquisition periods. Participation was voluntary, without incentive or disincentive, and responses were confidential without student identifiers. The internal Institutional Review Board approved the study prior to initiation. The participants had a traditional curriculum and a wellness center with access to counseling that had been established 2 years prior to matriculation, but no well-defined wellness curriculum or training for faculty on wellness.

\section{Study Measures}

Surveys included the seven-item, validated Medical Student Well-Being Index (MSWBI $)^{1}$ along with six demographic questions (age, gender, ethnicity, pathway to medical school-defined as traditional [directly from college] or nontraditional [college and medical school separated by time], work or research experience prior to medical school, and major life events within 6 months [marriage, divorce, birth of a child, death of a signficant friend or family member, or major illness or injury]). Figure 1 displays the survey.

\section{MSWBI}

The MSWBI ${ }^{1}$ is an instrument previously demonstrated to have reliability and strong content-related validity with high sensitivity and specificity for identifying severe distress in medical students. It measures five domains of distress (burnout, depression, fatigue, stress, QOL) with seven questions derived from several previously validated instruments. ${ }^{1,8}$ Table 2 denotes the distress domains, subdomains, and corresponding $\mathrm{MSWBI}^{1}$ questions in the column headers. The MSW$\mathrm{BI}^{1}$ was provided by the Mayo Clinic with a written agreement for its use.

\section{Severe Distress and Domains}

The MSWBI ${ }^{1}$ identifies students at risk of severe distress as measured by three clinically relevant outcomes: low mental QOL as measured by SF-8, suicidal ideation, and serious thoughts of dropping out of medical school. A threshold score of greater than or equal to four has comparable sensitivity and specificity to other validated screening instruments for identifying severe distress. ${ }^{8}$

\section{Burnout}

Previous studies have demonstrated the reliability of using single-item measures of emotional exhaustion and depersonalization, items which independently stratify the risk of burnout, for predicting key outcomes (ie, suicidality, decreased professionalism, and self-reported medical errors) in medical students. ${ }^{9}$ Questions one and two of the $\mathrm{MSWBI}^{1}$ independently correspond to emotional exhaustion and depersonalization, respectively. ${ }^{1}$ As such, we define students screening positive for burnout as having a positive response to at least one of question one or two.

\section{Statistical Analysis}

We have presented categorical variables by counts and percentages. We calculated proportions of respondents answering yes to each individual MSWBI ${ }^{1}$ question for each data acquisition period and compared for trend. We calculated the proportion screening positive for distress as the proportion of respondents answering yes to greater than or equal to four questions at a given data acquisition period. We calculated the proportion screening positive for burnout as proportion of respondents answering yes to either question one, two, or both for each acquisition period. We compared proportions for acquisition periods one (at orientation) and nine (after residency match) using standard $Z$ scores and assuming normal distribution. We determined $P$ values using a standard normal distribution table for conversion. We performed data analysis in Microsoft Excel.

\section{Results}

Students completed 886 surveys. The average class size was 120 students per acquisition period (1,047 in total). The average response rate was $85 \%$. Initial class of 2016 comprised $67(56 \%)$ male students, compared to $53.6 \%$ of matriculating medical students nationally, ${ }^{10} 76$ (63\%) Caucasian students compared to $51 \%$ nationally (in 2013), ${ }^{11}$ and age ranged from 20-32 years. At orientation, 63 (53\%) respondents were male students, 97 (86\%) respondents were 25 years old or less, 56 (47\%) respondents had entered medical school directly from undergraduate studies (traditional), and 13 (11\%) respondents had experienced major life events in the previous 6 months (either positive or negative events). The initial survey excluded ethnicity, but subsequent surveys included it. The class size was 114 students at graduation. Table 1 shows exact response rates per acquisition period along with proportion of male respondents to illustrate consistency of respondents at each acquisition. Demographics remained fairly constant through acquisition periods, except for periods three, six, and 
1. Do you feel burned out from medical school?*
a. Yes
No

2. Do you worry medical school is hardening you emotionally?*
a. Yes
No

3. During the past month, have you often been bothered by feeling down, depressed, or hopeless?
a. Yes No

4. In the past month, have you fallen asleep while stopped in traffic or driving?
a. Yes
No

5. During the past month, have you felt that all the things you had to do were piling up so high that you could not overcome them?
a. Yes
No

6. During the past month, have you been bothered by emotional problems (such as feeling anxious, depressed or irritable)?
a. Yes No

7. During the past month, has your physical health interfered with your ability to do your daily work at home and/or away from home?
a. Yes
No

1) Age - Circle one
a. $\leq 25$
b. $26-29$
c. $30-33$
d. $\geq 34$

2) Gender - circle one
a. Male
b. Female

3) Race/Ethnicity
a. Caucasian
b. African American
c. Hispanic
d. Native American
e. Asian
f. Other

4) How many years were between your undergraduate degree and entering medical school? Circle one
a. 0 (came straight from undergraduate to medical school)
b. 1
c. 2
d. $>2$

5) Have you had any other degrees, professions/jobs, or research experience before coming to medical school? You may circle more than one
a. Advanced degree after undergraduate school/college
b. Profession/jobs
c. Research
d. Other
e. None

6) Have you had any of these life experiences in the past 6 months? You may circle more than one
a. Birth of a child
b. Marriage
c. Divorce
d. Death of a significant friend/family member
e. Major illness/injury

* Questions 1 and 2 differed in the initial survey administered at orientation by reading:

1. Do you feel burned out coming into medical school?

2. Do you worry medical school will harden you emotionally? OR Do you feel hardened coming into medical school?

seven, which had response rates less than $85 \%$ and lower percentages of male students than the actual class. The sample size did not allow for meaningful comparison of distress domains amongst the demographic variables.

Table 2 shows prevalence for all seven distress subdomains, distress and burnout at each acquisition period. Depression and mental and physical QOL fluctuate at different acquisition periods, but prevalence from start to finish of medical school does not change significantly. Mental 
Table 1: Data Acquisition Periods

\begin{tabular}{|l|l|l|l|c|c|}
\hline $\begin{array}{c}\text { Acquisition } \\
\text { Period }\end{array}$ & $\begin{array}{c}\text { School } \\
\text { Year }\end{array}$ & \multicolumn{1}{|c|}{ Date } & \multicolumn{1}{|c|}{ Correlating Event } & $\begin{array}{c}\text { No. (\%) Total } \\
\text { Responses }\end{array}$ & $\begin{array}{c}\text { No. (\%) Male } \\
\text { Responses }\end{array}$ \\
\hline 1 & MS1 & Aug 2012 & Orientation & $113(94)$ & $63(53)$ \\
\hline 2 & MS1 & Nov 2012 & After initiating basic science classes & $108(91)$ & $57(48)$ \\
\hline 3 & MS1 & Apr 2013 & End of year 1 & $82(69)$ & $44(37)$ \\
\hline 4 & MS2 & Aug 2013 & After summer break & $111(94)$ & $60(51)$ \\
\hline 5 & MS2 & Nov 2013 & After initiating systems-based classes & $101(86)$ & $54(46)$ \\
\hline 6 & MS2 & Apr 2014 & After completing USMLE Step 1 & $94(82)$ & $49(43)$ \\
\hline 7 & MS3 & Oct 2014 & After initiating clinical clerkships & $76(67)$ & $41(36)$ \\
\hline 8 & MS3 & Apr 2015 & After standard year of clinical clerkships & $103(90)$ & $56(49)$ \\
\hline 9 & MS4 & Apr 2016 & After residency match & $98(88)$ & $56(50)$ \\
\hline
\end{tabular}

This table illustrates the specific periods of time that the research survey was administered to students, and response rates.

Table 2: Prevalence of Domains of Distress, Distress and Burnout by Acquisition Period

\begin{tabular}{|c|c|c|c|c|c|c|c|c|c|}
\hline $\begin{array}{l}\text { Acquisition } \\
\text { Period }\end{array}$ & $\begin{array}{c}\text { No. (\%) } \\
\text { Burnout } \\
\text { - EE }\end{array}$ & $\begin{array}{c}\text { No. (\%) } \\
\text { Burnout } \\
\text { - DP }\end{array}$ & $\begin{array}{c}\text { No. (\%) } \\
\text { Depression }\end{array}$ & $\begin{array}{l}\text { No. (\%) } \\
\text { Fatigue }\end{array}$ & $\begin{array}{l}\text { No. (\%) } \\
\text { Stress }\end{array}$ & $\begin{array}{c}\text { No. (\%) } \\
\text { QOL - } \\
\text { Mental }\end{array}$ & $\begin{array}{c}\text { No. (\%) } \\
\text { QOL - } \\
\text { Physical }\end{array}$ & $\begin{array}{l}\text { No. (\%) } \\
\text { Distress }\end{array}$ & $\begin{array}{l}\text { No. (\%) } \\
\text { Burnout }\end{array}$ \\
\hline Question: & \#1 & \#2 & \#3 & \#4 & \#5 & \#6 & \#7 & $\geq 4$ Yes & $\begin{array}{c}\text { Yes to } \\
\text { \# } 1 \text { or \#2 }\end{array}$ \\
\hline 1 & $6(5)$ & 15 (13) & $11(10)$ & $2(2)$ & 11 (10) & $42(37)$ & $3(3)$ & $2(2)$ & 19 (17) \\
\hline 2 & 17 (16) & 12 (11) & $27(25)$ & $3(3)$ & $27(25)$ & $47(44)$ & $6(6)$ & $6(6)$ & $27(25)$ \\
\hline 3 & $37(45)$ & 24 (29) & $29(35)$ & $3(4)$ & $39(48)$ & $49(60)$ & $21(26)$ & 24 (29) & 47 (57) \\
\hline 4 & 21 (19) & 30 (27) & $28(25)$ & $4(4)$ & $35(32)$ & $53(48)$ & $16(14)$ & $15(14)$ & $42(38)$ \\
\hline 5 & $28(28)$ & $25(25)$ & $24(24)$ & $5(5)$ & 27 (27) & $36(36)$ & 17 (17) & 16 (16) & $43(43)$ \\
\hline 6 & $21(22)$ & $26(28)$ & $40(43)$ & $1(1)$ & $43(46)$ & $66(70)$ & $6(6)$ & $19(20)$ & $33(35)$ \\
\hline 7 & $19(25)$ & 28 (37) & $26(34)$ & $2(3)$ & $21(28)$ & 36 (47) & $9(12)$ & $15(20)$ & $32(42)$ \\
\hline 8 & $45(44)$ & $43(42)$ & $41(40)$ & $6(6)$ & $41(40)$ & $57(55)$ & $13(13)$ & $33(32)$ & $61(59)$ \\
\hline 9 & $22(22)$ & $34(35)$ & 18 (18) & $4(4)$ & $25(26)$ & 36 (37) & $4(4)$ & $12(12)$ & 37 (38) \\
\hline \multicolumn{10}{|c|}{ Comparison of Acquisition Period 1 to Acquisition Period 9} \\
\hline$Z$ score & -3.66 & -3.67 & -1.82 & -1.01 & -3.04 & 0.07 & -0.58 & -3.05 & -3.44 \\
\hline$P$ value & $<0.01$ & $<0.01$ & 0.07 & 0.31 & $<0.01$ & 0.90 & 0.60 & $<0.01$ & $<0.01$ \\
\hline
\end{tabular}

This table denotes the prevalence of distress domains endorsed by students at each data acquisition period.

QOL is the worst at acquisition period 6, just after completion of USMLE Step 1, with $66(70 \%)$ respondents indicating poor mental QOL. Depression also peaks at this acquisition period at $40(43 \%)$ respondents. The overall prevalence of emotional exhaustion, depersonalization, stress, distress, and burnout significantly increase $(P<0.01)$ from orientation through graduation. Distress and burnout both peak at $33(32 \%)$ respondents and 61 (59\%) respondents, respectively, at the end of the year of standard clinical clerkships.
Figure 2 presents prevalence of emotional exhaustion, depersonalization, and burnout over 4 years. Emotional exhaustion peaks after the first and third years of medical school. Depersonalization increases throughout medical school with key inflection points at the end of year 1 and the end of the year of clinical clerkships.

\section{Discussion}

Implications of Findings

Medical students at the institution studied have similar demographic composition to other US medical schools and enter medical school with low prevalance of distress: two (2\%) respondents, by measure of a reliable screening tool. Depression and mental QOL fluctuate with stressful events in medical education, primarily, USMLE Step 1 (an intensive performance evaluation). Depression, mental QOL, and physical QOL all return to a previous baseline after a break or change in the stressors as evidenced by proportions experiencing these domains after summer break or after residency 
Figure 2: Prevalence of Burnout, Depersonalization, and Emotional Exhaustion Over Time

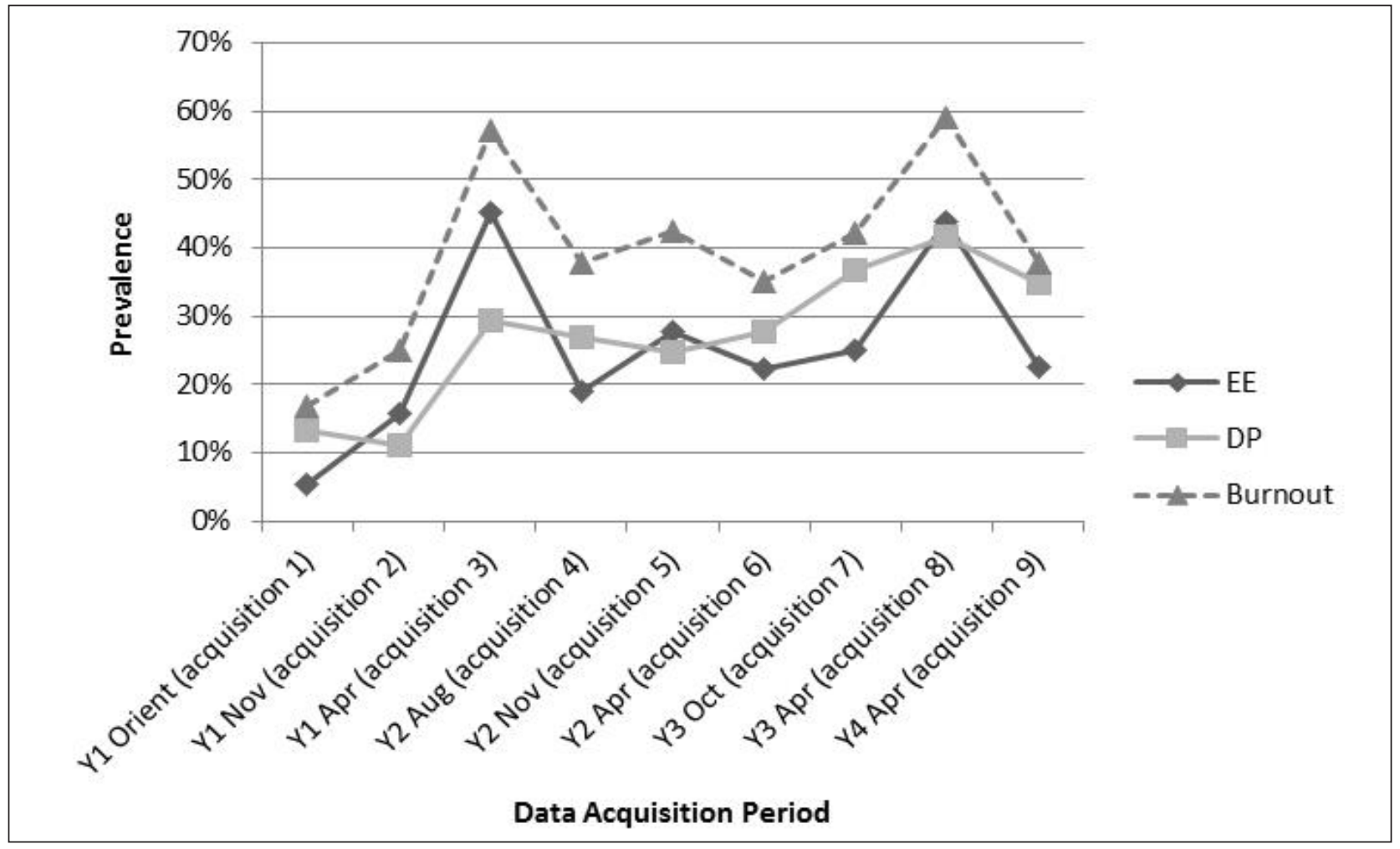

match. These trends suggest that medical student depression and low mental QOL are due, at least in part, to external mediators. This suggests that prevention and remediation are theoretically possible.

The data echoes prior studies demonstrating that students enter medical school with relatively low rates of burnout (17\%) and that burnout, including depersonalization and emotional exhaustion, develops during medical school. ${ }^{3,4}$ The major inflection points in distress and burnout occur at the end of year 1 and the end of the year of clinical clerkships. Large fluctuations in burnout appear to correlate with fluctuations in emotional exhaustion, while the overall trend appears to follow that of depersonalization as shown in Figure 2.

Depersonalization develops early in the educational process when students are focused on intensive study for preclinical coursework with multiple performance evaluations. Of note, the institution studied had a 5-point grading scale and a fairly traditional model of basic science and systems-based didactics with interspersed group sessions focused on clinical correlation, physical exam, and the doctor-patient relationship. Previous studies have postulated that preclinical coursework contributes to depersonalization and burnout. ${ }^{12}$ There is also an inflection point in depersonalization after completion of the year of clinical clerkships. Once depersonalization develops, it does not regress substantially, as evidenced by consistency prior to and after summer break, which appears to be a rejuvenating time for other distress domains. The permanency of depersonalization is alarming given the high association with decreased professionalism and prior evidence demonstrating that depersonalization created in medical school carries into residency. ${ }^{6,13}$ Prior studies have focused on preclinical interventions, including elimination of hierarchical grading schemes and introduction of self-care curriculum early in education. ${ }^{3,12}$ Our study supports the importance of early intervention to prevent the initial development of depersonalization, with continued intervention in the clinical clerkship year.

The finding in this study not emphasized in existing literature is the upward inflection of emotional exhaustion, and thus the peak of burnout that occurs at the end of the clinical clerkship year. Those of us who presume that clinical medicine is the regenerative part of the education process may need to recognize that our efforts to reform education should include a rethinking of the process of clinical education. The clinical block system of 30-40 years ago may be suboptimal for the learners of today. It is alarming that the initial exposure of medical students to their career of choice results in such distress. It is unclear if this is a new trend or simply new data representing an old trend. We encourage other institutions to apply the same methodology to determine the universality of this trend. An interesting future study would be contrasting the temporal trends in distress at multiple institutions. Maybe we can 
identify schools that consistently perform better and learn from them. If these trends appear universal, monitoring for changes in the trends with intervention studies could aid in development of new ways of teaching medicine.

\section{Limitations}

Surveys did not request student identifiers that would allow us to eliminate intersubject correlation as is necessary for true longitudinal analysis. We intentionally implemented this design to maintain anonymity, encourage participation, and elicit honest responses. The resulting data set provides sufficient data for trend monitoring at the cohort level.

A few data acquisition periods have lower response rates $(<85 \%)$ with samples that may inadequately represent the cohort as evidenced by the changes in the proportion of male respondents in Table 1. There is inherent population turnover in a single class of medical students due to attrition, combined degree programs, and other personal factors. Overall class size did not change greatly and authors postulate that this did not have a substantial impact on results.

Studies have validated the instrument utilized, MSWBI, ${ }^{1}$ as a screening tool, not a diagnostic measure for individual distress domains. Given that we surveyed the same cohort throughout the study, a screening tool is sufficient for monitoring trends. Single-item measures to determine emotional exhaustion and depersonalization, and therefore burnout, have demonstrated high sensitivity and specificity to measure burnout and its subdomains. ${ }^{9}$

\section{Conclusions}

Our study supports the literature by demonstrating the relatively low prevalence of distress and burnout at matriculation and the development of distress domains throughout medical education in a single cohort. Our contribution to the literature is the suggestion that depersonalization develops early with inflection points in both preclinical and clinical education without regression in prevalence once developed. Our data also brings attention to an upward inflection in emotional exhaustion associated with clinical exposure, which is not highlighted elsewhere in the literature.

As educators, we can improve the educational process and the "first impression" that medical students have of their future careers. In so doing, we hope to curtail the emotional exhaustion and depersonalization that ensue. Broader longitudinal studies are needed to determine if the trends observed at our institution are universal, with subsequent focus on studies to determine the most effective interventions at these key inflection points, with the ultimate goal of creating a healthier education environment.

ACKNOWLEDGMENTS: The authors would like to acknowledge the invaluable contributions of Stephen W. Davis, MA, as well as numerous students, including, Sara Glance, MD Rachel Conklin, PA, MPH, David Conklin, MD and Genni Kee, MD.

Presentations: A round table discussion of an abbreviated version of this article was presented at 2018 STFM Conference on Medical Student Education on February 3, 2018 in Austin, Texas.

CORRESPONDING AUTHOR: Address correspondence to Dr Maggie W. Hansell, Department of Family and Community Medicine, Wake Forest Baptist Health, Medical Center Blvd, Winston Salem, NC 27157. 336-713-6155. mhansell@wakehealth.edu.

\section{References}

1. Dyrbye LN, Szydlo DW, Downing SM, Sloan JA, Shanafelt TD. Development and preliminary psychometric properties of a well-being index for medical students. BMC Med Educ. 2010;10(1):8

2. Maslach C, Jackson SE, Leiter MP. Maslach Burnout Inventory Manual. 3rd ed. Palo Alto, CA: Consulting Psychologists Press; 1996.

3. Dyrbye L, Shanafelt T. A narrative review on burnout experienced by medical students and residents. Med Educ. 2016;50(1):132-149.

4. Brazeau CM, Shanafelt T, Durning SJ, et al. Distress among matriculating medical students relative to the general population. Acad Med. 2014;89(11):1520-1525.

5. Dyrbye LN, West CP, Satele D, et al. Burnout among U.S. medical students, residents, and early career physicians relative to the general U.S. population. Acad Med. 2014;89(3):443-451.

6. Dyrbye LN, Moutier C, Durning SJ, et al. The problems program directors inherit: medical student distress at the time of graduation. Med Teach. 2011;33(9):756-758

7. Ishak W, Nikravesh R, Lederer S, Perry R, Ogunyemi D, Bernstein C. Burnout in medical students: a systematic review. Clin Teach. 2013;10(4):242-245.

8. Dyrbye LN, Schwartz A, Downing SM, Szydlo DW, Sloan JA, Shanafelt TD. Efficacy of a brief screening tool to identify medical students in distress. Acad Med. 2011;86(7):907-914.

9. West CP, Dyrbye LN, Satele DV, Sloan JA, Shanafelt TD. Concurrent validity of singleitem measures of emotional exhaustion and depersonalization in burnout assessment. J Gen Intern Med. 2012;27(11):1445-1452.

10. Association of American Medical Colleges. FACTS -Applicants, First-Time Applicants, Acceptees, and Matriculants to U.S. Medical Schools by Sex, 2007-2008 through 2016-2017. https://www.aamc.org/download/321470/data/ factstablea7.pdf. November 21, 2017. Accessed January 18, 2018.

11. Association of American Medical Colleges. FACTS -Table A-14.2: Race/Ethnicity Responses (Alone and in Combination) of Matriculants to U.S. Medical Schools, 2013-2014 through 2017-2018. https://www.aamc.org/ download/485288/data/2017factstablea14_2. pdf. November 30, 2017. Accessed January 18, 2018.

12. Reed DA, Shanafelt TD, Satele DW, et al. Relationship of pass/fail grading and curriculum structure with well-being among preclinical medical students: a multi-institutional study. Acad Med. 2011;86(11):1367-1373.

13. Dyrbye LN, Massie FS Jr, Eacker A, et al. Relationship between burnout and professional conduct and attitudes among US medical students. JAMA. 2010;304(11):1173-1180. 